\title{
Aspects of the extension of forty exploitation of bulk reservoirs for irrigation and hydropower purposes
}

\author{
Dilshod Bazarov ${ }^{1 *}$, Bobur Shodiev ${ }^{1}$, Boxodir Norkulov ${ }^{2}$, Umida Kurbanova ${ }^{2}$ and Boyburi \\ Ashirov $^{3}$ \\ ${ }^{1}$ Tashkent Institute of Irrigation and Agricultural Mechanization Engineers, Waterpower exploitation \\ and pumping stations, 100000, Tashkent, Kara-Niyaziy str, Uzbekistan. \\ ${ }^{2}$ Samarkand State Architectural Building Institute, 140100, Samarkand, Lolazor str., Uzbekistan. \\ ${ }^{3}$ Karshi State Engineering and Economic Institute, 180100, Karshi, Mustakillik 225 str., Uzbekistan.
}

\begin{abstract}
The article is devoted to solving the problems of reducing the useful volume of bulk reservoirs, which is an urgent task of water management. The development of the design allows to reduce the volume of incoming sediment into the reservoir thicket is the main goal of the present work. The analysis of materials of field studies and a review of existing scientific works was the basis of the research method. The results of field studies on the study of channel processes in the downstream of the feeder channel of a low-pressure reservoir and the dynamics of their siltation. It has been established that the reservoirs accumulate only $0.3-10 \%$ of the annual river flow and the degree of annual silting of the capacity of such reservoirs is on average $0.5-2.0 \%$. After $25-50$ years, reservoirs may lose half of their usable capacity and after 40-80 years, a complete failure of operation is possible. To slow down this process in the basins of the self-leveling reservoirs, a new structure of structures developed, which ensures an even flow of water in the supply channel and maximum sedimentation of sediments in the channel, which prevents them from entering the reservoir bowl.
\end{abstract}

\section{Introduction}

When water enters the reservoir, a sharp change in the hydrological and hydraulic flow regimes occurs. Since it is typical for the reservoirs of our republic to redistribute the flow of the river over time - the accumulated part of the flow during the non-growing season, some undesirable processes occur in terms of operation to supply this volume at the required time. For example, water flows into the reservoir bowl with a significant amount of sediment $\left(12-17 \mathrm{~kg} / \mathrm{m}^{3}\right.$ or more), as a result of a sharp decrease in the average flow velocity, the majority of these sediments are deposited in the reservoirs. Since the reservoirs accumulate only $0.3-10 \%$ of the annual river flow and the degree of annual silting of the capacity of such reservoirs is on average $0.5-2.0 \%$. In 25-50 years they can lose half of their usable capacity, which means their withdrawal, and in $40-80$ years $[1,2,3]$.

\footnotetext{
* Corresponding author: dr.bazarov@ mail.ru
} 
Many of the reservoirs in the region are classified as liquid, where water is supplied with supply channels originating from natural watercourses. Very often, due to the large differences in terrain marks, a connecting structure is projected along the canal. The interfacing construction in hydraulic engineering or hydropower construction are designed for emergency discharge of water into the lower reach or on short sections of rivers and canals that have a culvert with a large difference in elevations. These structures are built in the form of a differential or short channel with a high slope - rapid current [2,3]. At the entrance of the connecting structure from the spill-over threshold and within the anchorage, the flow has a sufficiently large excess kinetic energy, much of which is spent on reshaping the bed and coast, within the downstream anchorage $[15,16,17,18]$.

Despite numerous studies of various types of damping devices, as well as evaluations of their impact on flow structure and the ability to control faulty currents, reducing their undesirable effects on the mount and the natural course, many of the issues noted above are not fully understood, the results obtained in these studies are often contradictory, and sometimes are mutually exclusive [3,4]. Based on the above, the purpose of this work is defined, which consists of developing hydraulic structures on the basis of hydraulic calculations to prevent sediment from entering the reservoir thicket and destroying the spillway structure in the downstream of the low-pressure hydraulic system, and propose a method for calculating this structure which will help to prevent undesirable channel deformations of the outlet channel bowl.

\section{Research methodology}

For studying and analyzing the current channel processes, materials from field studies to determine the decrease in the useful volume of the reservoirs [1] of irrigation and hydropower purposes and the results of studies conducted on a specific object $[5,6]-$ the supply channel of Aktepe reservoir were used. The territory of Aktepe reservoir is located on the left bank of the Surkhandarya River, $30 \mathrm{~km}$ north of the city of Termez, and is a depression extending from north to south, 7-7.5 km long, 3-4 km wide. From the west it is bounded by the Amu-Zang and Kokaity canals, from the north and east by the foothills of the Ak-Tau range.

The slope of the groundwater mirror $\mathrm{I}=0.01$ in some areas increases to 0.05 . The end section of the trapezoidal feed channel in a concrete lining, $2 \mathrm{~km}$ long, with zigzag ribs of artificial roughness. The channel is designed to pass $25 \mathrm{~m} 3 / \mathrm{s}$. [5,6]

\section{Research and discussion results}

Due to the high saturation of the flow, the silting dynamics of the reservoirs entering the reservoir thicket is of an intensive nature (Table 1).

Table 1.

\begin{tabular}{|c|c|c|c|c|c|c|}
\hline № & $\begin{array}{l}\text { Name of bulk } \\
\text { reservoirs }\end{array}$ & $\begin{array}{c}\text { Water } \\
\text { abstraction }\end{array}$ & $\begin{array}{l}\text { Gross } \\
\text { capacity, } \\
\operatorname{mln}^{3} \mathrm{~m}^{3}\end{array}$ & $\begin{array}{l}\text { Operati } \\
\text { ng } \\
\text { period }\end{array}$ & $\begin{array}{c}\text { Silting } \\
\text { dynamics } \\
\text { during the } \\
\text { operating } \\
\text { period }\end{array}$ & $\begin{array}{l}\text { Silting } \\
\text { capacity }\end{array}$ \\
\hline 1 & Akdaryo & Akdarya & 112.5 & 20 & 93.17 & 17,18222 \\
\hline 2 & Andijan & Kharadarya & 1900 & 20 & 1664.8 & 12,37895 \\
\hline 3 & Akhangaran & Akhangaran & 210.36 & 24 & 200.16 & 4,848831 \\
\hline 4 & Jizzax & Sanzor & 100 & 30 & 80.08 & 19,92000 \\
\hline 5 & Kattakurgan & Zarafshan & 900 & 35 & 697.46 & 22,50444 \\
\hline
\end{tabular}




\begin{tabular}{|c|c|c|c|c|c|c|}
\hline 6 & South Surkhan & Surkhandarya & 800 & 35 & 503 & 37,12500 \\
\hline 7 & Quemazar & $\begin{array}{l}\text { Amudarya, } \\
\text { Zarafshan }\end{array}$ & 310 & 42 & 274.5 & 11,45161 \\
\hline 8 & Pachkama & Guzardarya & 260 & 36 & 192.6 & 25,92308 \\
\hline 9 & Talimarjan & $\begin{array}{c}\text { KMK, } \\
\text { Amudarya }\end{array}$ & 1525 & 17 & 1464.03 & 3,998033 \\
\hline 10 & Tashkent & Angren & 250 & 37 & 207.68 & 16,92800 \\
\hline 11 & Tudakul & $\begin{array}{l}\text { ABMK, } \\
\text { Zarafshan }\end{array}$ & 1200 & 33 & 1035.4 & 13,71667 \\
\hline 12 & Tupalang & Tupalang & 100 & 20 & 83.35 & 16,65000 \\
\hline 13 & Aktepe & Amudarya & 100 & 13 & 89,27 & 9,730000 \\
\hline
\end{tabular}

It should be noted that only suspended sediments transported by water flow from the river enter the bulk reservoirs and they can often flow through the reservoir along with the stream, and if we take into account the fact that during the filling of the bulk reservoirs (during the winter months and before the growing season) the average turbidity of the rivers of Central Asia) is the minimum value $\left(0.5-4 \mathrm{~kg} / \mathrm{m}^{3}\right)$, then it can be determined that the reservoirs will be silted annually by 0.002-0.004 part of the useful capacity of the reservoir for their full siltation the storage will take several hundred years $[1,7,8]$. Usually, in many rivers, measures ensuring the prevention of the flow of bottom and coarse sediment fractions are carried out at the head water intake structure on the river and they, as a rule, do not enter the reservoirs. On the rise of the flood, when the flow carries the greatest amount of sediment and at the same time the flow of the river is still small, it is possible to regulate the volume of water supplied to the bulk reservoirs, thereby reducing the volume of their sedimentation $[9,10]$.

As shown by the results of research in the reservoirs of the Amudarya and Syrdarya river basins, the design period for the sedimentation of the dead volume of these reservoirs varies from 34 to 126 years and the silting period for the total volume of the reservoir is more than 700 years. The average value of the annual loss of the capacity of the bulk reservoirs is $0.11 \%$, therefore, the sedimentation of such reservoirs mainly occurs only at the expense of mudflows. The annual solid flow of such rivers is $0.03-0.7 \%$ of the reservoir capacity, i.e. their full silting will occur after 150-1000 years of operation of the reservoir [3,7].

Analysis of the operational services data, following the course of re-formation of the channel at the dams of the channel reservoirs located in our state with a capacity of more than 50 million $\mathrm{m} 3$, from the beginning of their operation to the present time showed that the main factor in reducing the useful volume of the reservoir is the deformation of the channel of the reservoir. This is due to the sedimentation of sediment entering the reservoir along with the stream. As a result of a sharp expansion of the channel in the inlet, the average velocity decreases, which leads to a decrease in the transporting capacity of the stream, contributing to the beginning of the silting process. The actual volume of sediment deposited in the reservoir always exceeds the design volume, (Table No. 2):

Table 2.

\begin{tabular}{|c|c|}
\hline Name of bulk reservoir & $\begin{array}{c}\text { Exceeding capacity from } \\
\text { designed capacity }\end{array}$ \\
\hline Tuyamuen & 1,5 \\
\hline Kayrakkum & 1,5 \\
\hline Tashkent & 1,5 \\
\hline South surkhan & 1,5 \\
\hline Andijan & 1,8 \\
\hline Chimkurgan & 4,7 \\
\hline Akkhangaran, Pachkamar & $6,2-7,2$ \\
\hline
\end{tabular}




\begin{tabular}{|c|c|}
\hline Talimarjan,Kattakurgan, Kuemazar & $1,25-2,00$ \\
\hline
\end{tabular}

As the analysis of the deformation processes, the dynamics of the change in the volume of incoming nans depends on the location of the reservoir, if the reservoir is lower, i.e. is located in a low mark to it, a large number of nanos will be performed, otherwise the number of incoming nanos in the reservoir will be much smaller. In addition, the reduction of the useful volume of the reservoir is contributing to reduce the intensity of the hill level. 7.] To study the operational state of the supply channel, scientific studies were carried out in the channel of the supply channel of the acting of the pushing reservoir [5,6]. As a result of the studies, it was found that the earth's section of the supply channel has waste and deformation (Fig. 1).
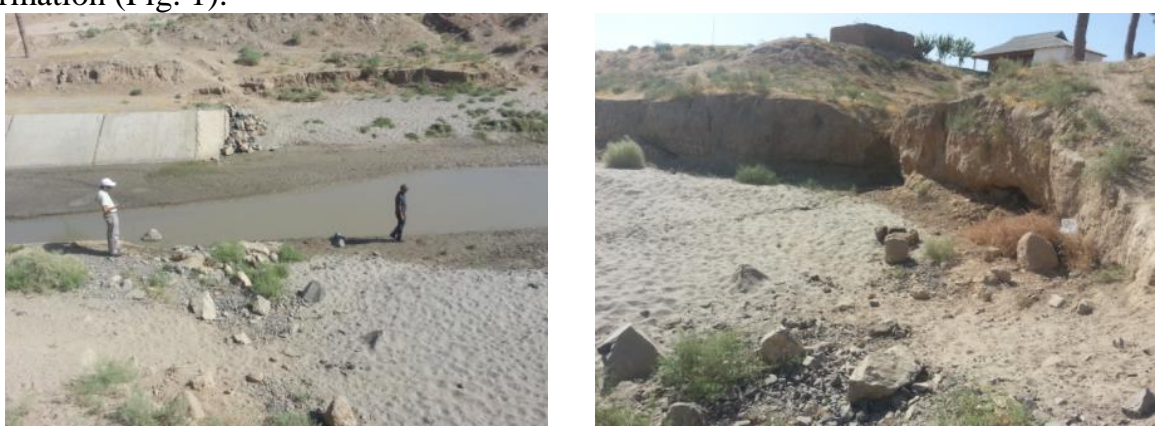

Fig. 1. Deformation processes behind the concrete part of the supply channel

This is the absence of the flow after the concrete section of the supply channel. Because of the high flow rates and its slums, there was an intense coastal deformation from the above channel of the channel [18]. In the mouth of the supply channel, intensive wetlands have been made in concrete slabs, which contributed to the appearance of a positive transverse slope. There are places with destroyed ribs of the gas into the concrete linen of the channel of the channel. The expanding concrete part of the channel interface from the bowl of the reservoir has destruction. As a result, zigzagic carriers began to be destroyed and the destruction of the bottoms and the shores began to be destroyed (Fig. 2).
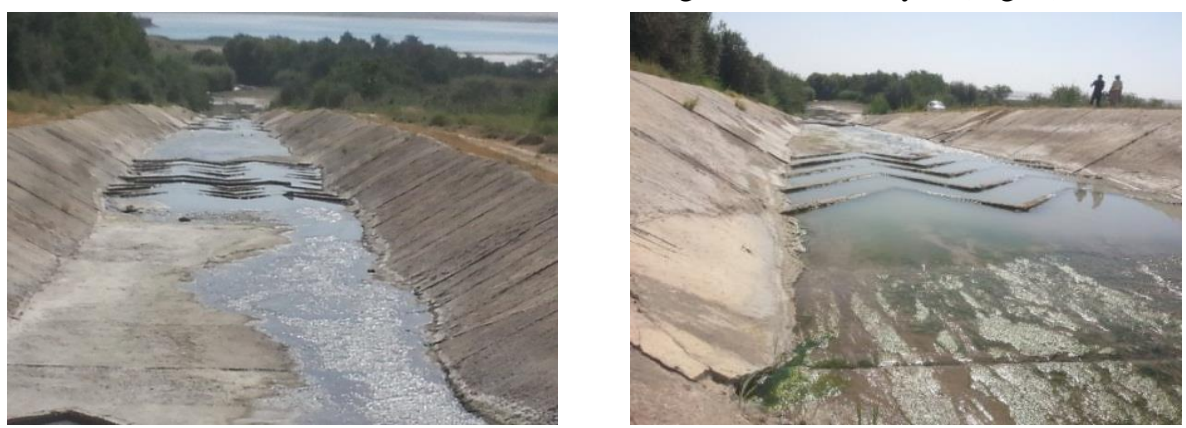

Fig. 2. Deformations in the zone of fastening of the mouth of the supply of channel

The bowl of the reservoir is provided by a smoothly expanding site. There are places with destroyed ribs of gas on the concrete lining of the channel of the channel. The expanding concrete part of the channel interface with the bowl of the reservoir has destruction (Fig. 3). 

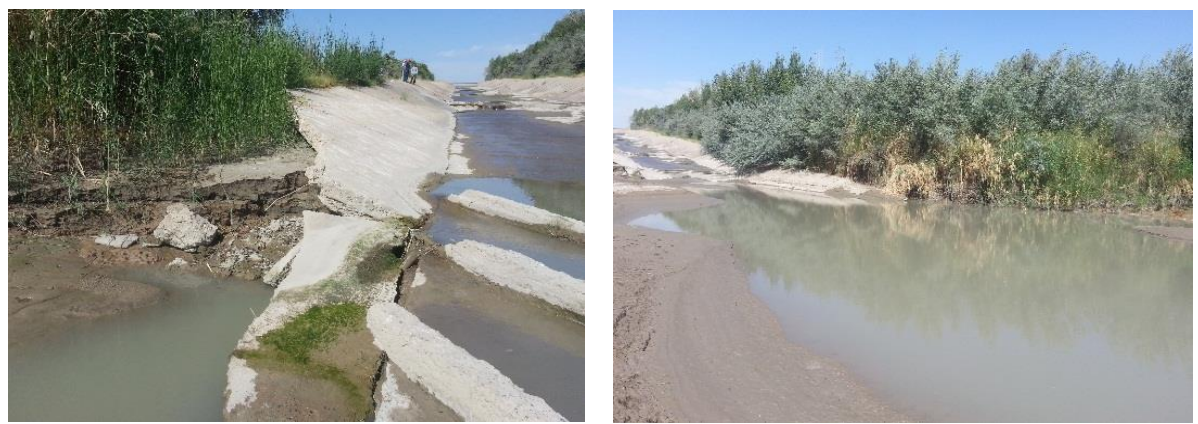

Fig. 3. Deformation processes at the interface of the inlet channel with the reservoir basin

In addition, the reservoir bowl is intensively silted due to incoming sediment. This contributes to an intensive decrease in its usable volume, a decrease in useful volume over 13 years of operation was $9 \%$. (Table number 1 ).

For the operating conditions of the supply channel of the reservoir and the extension of the service life, a design has been developed that allows reducing the amount of incoming sediment into the reservoir bowl. The design contains spillway structures located perpendicular to the channel of the supply channel, elements of the interfacing structures, the nanostorage and side outlets to the nanostorage. The nanostorage has a mark below the bottom of the supply channel and above the maximum water level in the reservoir and a wall of pebbles of large dimensions that perform the filter function of residual turbid water in the channel of the channel. The road for trucks and equipment for its seasonal cleaning is provided in the bowl of the depository.

Since the supply channel has a high bottom slope, which contributes to the emergence of high flow rates during the passage of the design flow, during the reservoir filling in the channel there will be a suction mode. This will lead to intensive planned and deep deformations and intensive silting of the reservoir bowl due to the product being eroded [2, $10,11]$.

To solve the mentioned problem in the channel of the supply channel, it was recommended to reconstruct it with the construction of interfacing structures at the interface of the concrete part with the earthen channel in the initial section and in the mouth of the channel with the following scheme and hydraulic parameters [11,12,13,14-21] (Fig .four).

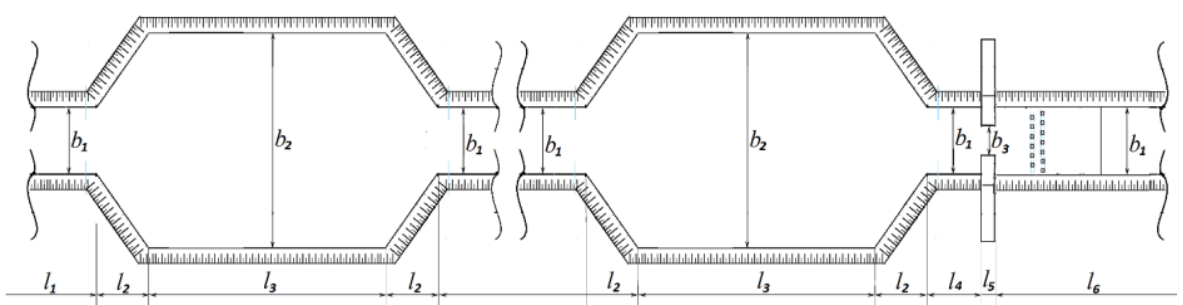

Fig. 4.

The calculation scheme of the supply channel of the acting reservoir to ensure the formation of a uniform motion mode and the conditions for the complete deposition of the water streams of the water flow in the channel of the supply channel, it is necessary to build a bracinging structure with a trapezoidal water pipe and a watermeter at its final section $(1.7 \mathrm{~km})$ with the following structure [18] (Fig. 5): 


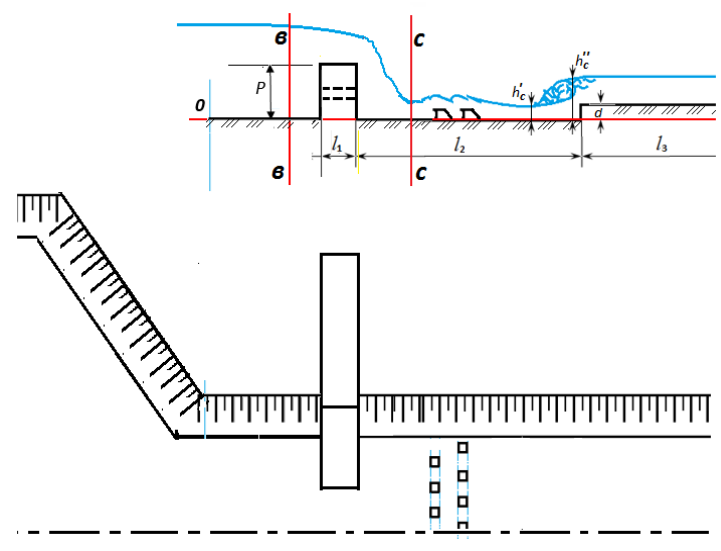

Fig. 5.

Recommended scheme of the supply channel after the trapezoidal mutual-adapted the calculated parameters were as follows: $\mathrm{P}=2 \mathrm{~m}$-is the difference between the marks of the upper and lower beef; $l_{1}=0.5 \mathrm{~m}$-is the width of the trapezoidal multimode; $b_{1}=3 \mathrm{~m}$-is the width of the subflution channel; $m=1$ - is the width of the submarine; the calculated values of the mandatory structures of the supply channel were the following: $d=0.44 \mathrm{~m}$ is the depth of the waterfall well; $h_{c}^{\prime}=0.37 \mathrm{~m}$ is the first conjugate depth of the hydraulic jump; $h_{c}^{\prime \prime}=1.55 \mathrm{~m}$ - the second conjugate depth of the hydraulic jump $l_{2}=l_{\text {в.к. }}=5.18 \mathrm{~m}$ - the length of the waterfall well; $l_{3}=l_{n . n}=46.5 \mathrm{~m}$ - the length of the post-length section of the renewal of the reservoir of the reservoir is proposed to introduce a design, which allows to reduce the number of incoming nanos in the bowl of the reservoir, the structure will contain spiders of the reservoir structures, performed the perfume of the water supply to the nanosolus structure, the nanososter and side of the water supply to the nanososurus, the nanososter, the naval and the side of the water and the above-mentioned channel and the above-mentioned channel of the maximum water level in the reservoir and the abovementioned channel of the midwily the function of the filtrate of the residual flour water in the channel of the channel, the filling the filling the fan residue of the channel with the fan of the channel.

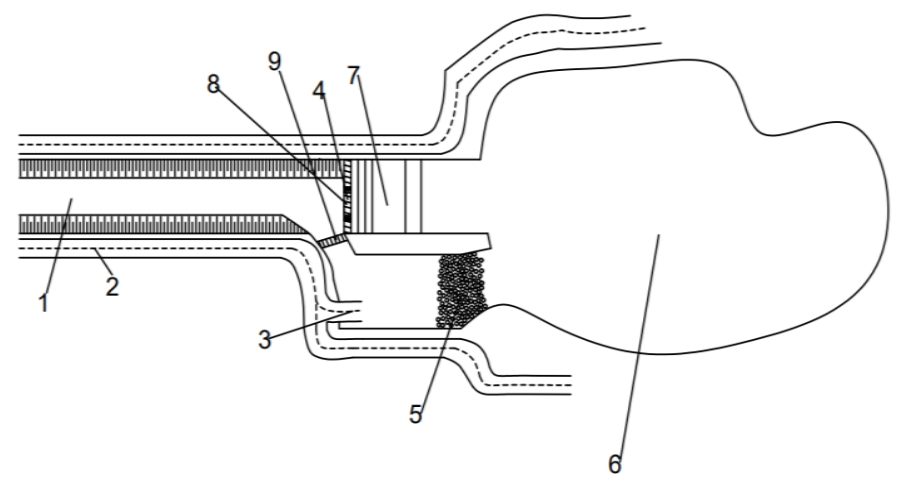

Fig. 6. The proposed scheme of the nano conservation complex:

1 - Supply canal, 2 - Road , 3 - Mouth of nano conservation, 4-Check, 5 - Gravelly wall, 6 -

Reservoir, 7 - Conjugation structure, 8 - Spillway gate with wide rapids, 9 - Water gate 
As above is noted, the input of the conjugate part of the binding channel is provided with a landing channel. The earthly side of the banding channel is exhausted with average speed of the water supply with an average speed of the second-hand type of medium speed with the average speed of the second-hand type of medium speed with the average speed of the second-hand type of medium speed, the medium speed is less than the tapping of the flow of the flow along the flow of the water with an average speed less than the tapping flow of the flow along the flow of the flow of the flow of the flow of the geometric dimension of the channel, the device is achieved by changing the geometric dimensions of the channel, and the device of the transitional-burning structure at the end part channel. The overhead structure is provided with a puff, the dash of the insert structure (4) to the natives the market, the bottom of the internal structure is used below the bottom of the water and the level of the influenza structure, the base, the installation is used in the closed position for the deposit of the reservoir, the blade is placed in the closed position for the reservoir, the shutter, the conservation plaque was used, the puncture is used to the filler structure, the constructing line, the concrete structure was used for the filler structure, the conventional route from the stone and 2] for the specialties.

The device for comprehensive control of the navigation movement in the supply channel of small reservoirs works as follows: during the reservoir filling in the supply channel (1), the water passage mode is obtained with a minimum permissible speed that is less than not the hyping flow rate. The formation of such a mode is uneven. The clarified flow is passed through the overcoming structure, the pulmonary with a wide threshold, the difference and the matching plant is almost much lower than the height of the threshold of the puff. The maximum level of the shutter's rapid mounted in the inlet lane of the nanosojstra is the lower threshold. The water flowing passing the powder. The water flow passing through the height of the threshold. The water flowing passing the mid-loss and through the conjugate section in the channel of the lower beech moves to a uniform traffic mode with an average speed less than a non-blowing flow rate of the flow, the binding structures is reduced by the uniform modal of the water flow to the bonds of the reservoir, the supply channel is provided by the factor of the water supply of the water supply to the reservoir, the closure is installed on the surface of the deposits, the simultaneous opening of the pumping plane, the shutter structures are carried out into the naval the stage, the channel will be operated in the spot of water to the nanososser, the channel will be carried out in the stream of water to the nanososser, the channel, the channel is powered by the water flow in the nanososser, the canvas streams in the place with the flow of water to the nanosossery can be transported in the naval stage.

\section{Conclusions and recommendations}

The following conclusions were made:

1.For improvements of the operating conditions of the supply channel must be reconstructed;

2.To overcoming the life of the reservoir must be implemented to reduce the number of incoming nanos in the bowl of the reservoir. The design will contain spiders of the supply of the supply, the elements of the matching structures, the nanososcan and side water to the nanosure. The nanos has a mark below the bottom of the supply channel and above the maximum water level in the reservoir and the wall of the large maximum water levels of the residue of the residual mutal water in the channel of the large dimensions that perform the function of the filter of residual mutumous in the channel of the range. The band of the fundamental mutal water in the channel of the channel can be used in the band of the pan. The compositions are used to compare the mating the structures for the construction of a 
concrete pipeline for the trucks and the equipment for the implementation of its seasonal cleaning;

3. It is necessary to build interfacing structures on the section of interface of the concrete part with the earthen channel in the initial section of the channel and the mouth of the supply channel;

4. According to the results of hydraulic calculations, the calculated parameters are taken: $P$ $=2 \mathrm{~m}$ - the difference between the bottom and bottom pool marks; $l_{1}=0.5 \mathrm{~m}$ - the width of the threshold of the trapezoidal weir; $l_{2}=l_{\text {k.K. }}=5.18 \mathrm{~m}$ - the length of the water well; $l_{3}=l_{n . n \text {. }}$ $=46.5 \mathrm{~m}$ - the length of the post-jump area; $b_{1}=3 \mathrm{~m}$ - the width of the supply channel; $b_{2}=$ $10 \mathrm{~m}$ - the width of the sump; $m=1$ is the slope coefficient of the supply channel; $d=0.44$ $\mathrm{m}$ is the design depth of the water well; $h_{c}^{\prime}=0.37 \mathrm{~m}-$ the first coupled depth of the hydraulic jump; $h_{c}^{\prime \prime}=1.55 \mathrm{~m}$ - the second coupled depth of the hydraulic jump. The estimated length of the mounting area is recommended to be taken equal to $53.9 \mathrm{~m}$.

The authors of this scientific work express their sincere gratitude to the administration of the Tashkent Institute of Engineers of Irrigation and Agricultural Mechanization, represented by the rector - Doctor of Economic Sciences, Professor Uktam Pardaevich Umurzakov for the opportunity during the review in the scientific library, created a cozy atmosphere for writing this scientific work, and financial support until publication. And also, the authors consider it their duty to particularly note the great help of the staff of the Amu-Zang Irrigation Systems Department of the Ministry of Water Resources of the Republic of Uzbekistan, who presented the opportunity to conduct full-scale studies and get acquainted with the materials of full-scale studies of the Aktepe reservoir feed channel.

\section{References}

1. Ahmedkhodjaeva I.A. "Methods for predicting the loss of capacity of channel reservoirs of seasonal regulation". Diss. For the degree of Ph.D. (2008)

2. Baryshnikov NB, Isaev D. Channel processes. St. Petersburg. Ed. RGTMU, 459 (2014)

3. Bazarov D.R., Arafjanov ...etc. Processes in the lane, T. TIQXMMI, 641 (2018)

4. Baryshnikov NB Flow dynamics. St. Petersburg. Ed. RGTMU, 439 (2007)

5. Bazarov D.R., Khidirov S.K. Recommendations for the prevention of channel deformations in the downstream pools of reservoirs. MAAF Tashkent, 45 (2017)

6. Bazarov D.R. and others. NTO on x / d on the topic №17 / 2017 "Development of recommendations for the prevention of channel deformations in the downstream pools of reservoirs" MAWR, Tashkent, 80 (2017)

7. Avakyan A.B. and others. Reservoirs. - M .: Thought, (1987)

8. Skrylnikov V.A. "Methods of calculating the elements and modes of operation of the main supply of open irrigation systems" Thesis for the degree of D.Sc. T. (1996)

9. Raskazov L.N. et al. Hydraulic Structures, Part I, Due to the Association of Building Institutions, M. 574 (2008)

10. Raskazov L.N. et al. Hydraulic Structures, Part II, Due to the Association of Building Institutions, M. 527 (2008)

11. Bazarov D.R., Karimov R.M., Hidirov S.Q., Matyakubov B.SH. GIDRAVLIKA II SPECIAL COURSE, TIQXMMI, 555 (2018)

12. Bazarov D.R., Karimov R.M., Hidirov S.Q., Matyakubov B.SH. GIDRAVLIKA I BASIC COURSE, TIQXMMI, 526 (2018)

13. Zuykov A.L. Hydraulics Volume 1 M., due to the TSF NRU MSSUB 456 (2016)

14. Zuykov A.L. Hydraulics Volume 2 M., due to the TSF NRU MSSUB 423 (2016) 
15. Bazarov D.R., Khidirov S.K. Analysis of methods for assessing the hydrodynamic effects of flow on fasteners for hydrodynamic structures. "Architecture, Building, Design" 2, 2011. Ed. TASI, Tashkent, (2011)

16. Bazarov D.R., Khidirov S.K. Analysis of existing methods for calculating the attachment of downstream hydraulic structures for stability and durability. "Architecture, Kurilish, Design” 1, 2012 Ed. TASI, Tashkent, (2012)

17. Chernykh ON, Komelkov L.V. Hydrodynamic loads and stability of fixing the downstream of hydraulic structures // Hydraulic engineering., №8 24-28 (1983)

18. Khidirov, S.K., Norkulov, B. Analysis of methods of calculation of deformational processes in reservoirs bottom. The XVI Young Scholars, Masters and gifted Students' Scientific Conference on "Modern Problems in Agriculture and Water Management" TIҚHMMI Tashkent. 357-359 ( 2017)

19. Rasuanandrasana Marie Josefen. Hydraulic substantiation of the methods of calculation and design of the end sections of the pressure culverts with a vertically ascending flow output. Thesis for the degree of candidate of technical sciences in the specialty 05.23.16; 05.23.07. Hydraulics and engineering hydrology, Moscow, 170 (2010)

20. Nikolaenko, Yu.M. Substantiation of technical solutions for culverts taking into account the features of hydraulic regimes in operating conditions. Thesis for the degree of Doctor of Technical Sciences in the specialty of hydraulic engineering and land reclamation construction. Saint Petersburg. 386 (2000)

21. Kaveshnikov A.T. Features of the calculation and design of the elements of the outlets of waterworks. Thesis for the degree of doctor of technical sciences.Moscow. 411 (1993) 